\title{
Endoscopic management of esophageal leaks
}

\author{
Gabie K. B. Ong ${ }^{1}$, Richard K. Freeman ${ }^{2}$ \\ ${ }^{1}$ Department of Surgery, ${ }^{2}$ Division of Thoracic and Cardiovascular Surgery, St. Vincent Hospital, Indianapolis, Indiana, USA \\ Contributions: (I) Conception and design: All authors; (II) Administrative support: All authors; (III) Provision of study materials or patients: All \\ authors; (IV) Collection and assembly of data: All authors; (V) Data analysis and interpretation: All authors; (VI) Manuscript writing: All authors; (VII) \\ Final approval of manuscript: All authors. \\ Correspondence to: Richard K. Freeman, MD, MBA. 8433 Harcourt Road, Indianapolis, Indiana 46260, USA. \\ Email: Richard.Freeman@StVincent.org.
}

\begin{abstract}
Traditionally, gold standard treatment for an acute esophageal perforation has been operative repair. Over the past two decades, there has been a paradigm shift towards the use of esophageal stents. Recent advances in biomaterial allowed a new generation of stents to be manufactured that combined (I) a non-permeable covering; (II) radial force sufficient to occlude a transmural esophageal injury and (III) improved ease of removability. The amalgamation of these developments set the stage for utilizing esophageal stents as part of the management algorithm of an acute esophageal perforation. This provides a safe and less invasive treatment route in lieu of direct primary repair and its well-documented significant failure rate. Esophageal stent placement for failed operative repair or esophageal leaks also had the potential to minimize the need for esophageal resection and diversion. When included in a multimodality hybrid treatment protocol, esophageal stents can optimize healing success rates and minimize the risks of adverse complications. This review summarizes the modern history of esophageal stent use in the treatment of esophageal perforation as well as the evidenced based recommendations for the use of esophageal stent placement in the treatment of acute esophageal perforation.
\end{abstract}

Keywords: Esophageal perforation; esophageal fistula; esophageal stent

Submitted Sep 01, 2016. Accepted for publication Jan 20, 2017.

doi: $10.21037 /$ jtd.2017.03.100

View this article at: http://dx.doi.org/10.21037/jtd.2017.03.100

\section{Introduction}

Historically, utilizing an endoluminal esophageal stent to treat esophageal stenosis, fistulae and leak is not a new concept for the thoracic surgeon. The first successful experience was described by Symonds in 1887 using prostheses made of ivory and silver (1). In 1914, Guizes was the first to place esophageal "tubes" under direct visualization to palliate esophageal obstructions (2). Ten years later, Souttar published his results using metallic tubes with a rubber funnel (3). Coyas subsequently designed a plastic tube with metallic rings of equal diameter which was better tolerated by patients with malignant dysphagia (4).

In more recent times, Mousseau, Atkinson and Ferguson all developed devices for esophageal intubation $(5,6)$. Celestin, modifying a French design by Mousseau and Barbin, developed a polythene stent for inoperable malignant strictures that was successful in maintaining oral intake (7). However difficulty with insertion, migration and extraction limited the use of these prostheses.

With engineering and technological advancements, selfexpanding metallic esophageal stents became available in the 1990's based on techniques used to manufacture endovascular stents. These stents are woven, knitted or laser-cut metallic mesh designed to exert self-expansive forces up to a fixed diameter. The metallic part is most often a steel alloys such as elgiloy or nitinol. Elgiloy (cobalt, nickel and chromium) is corrosion resistant and able to generate high radial pressures, while Nitinol (nickel and titanium) allows more flexibility with less radial forces $(8,9)$.

Self-expanding metal stents offered multiple advantages including being inserted with a flexible esophagoscopy, 
significantly less esophageal dilatation, a lower rate of migration and improved palliation for malignant esophageal strictures and malignant tracheo-esophageal fistulae $(10,11)$. However, placement of these prostheses continued to be reserved only for the patient requiring palliative therapy for a malignancy because of the potential esophageal damage associated with extraction including reports of irreparable, sometimes life threatening, fistulae.

To combat these complications, second generation metallic stent designs were developed incorporating a covering of silicone, polyurethane or other polymers (12). While offering the advantage of diminishing amount of tumor ingrowth and fixation to the esophageal wall, it came with the cost of higher migration rates. Later, the ends of these covered stents were modified to be partially covered with a margin of $1.5 \mathrm{~cm}$ on both proximal and distal ends to optimize purchase of the esophageal wall (13).

Further evolution in esophageal stent biomaterials resulted in the development of an occlusive plastic prosthesis coated with silicone. This new design allowed ease of insertion, a minimal requirement for esophageal dilation and the ability to form an occlusive seal within the lumen of the esophagus (14). The distinctive advantage of these non-metallic endoprostheses was the ability for removal or replacement even after an extended period of time without damage to the esophagus. However, similar to the covered metallic stents, a higher incidence of migration was appreciated (15).

These synergistic developments led to a crucial turning point in management of esophageal perforations. The ease in manipulation of high-quality covered, occlusive stents in the esophagus, led some investigators to implant these stents in select patients as a temporary measure to treat intrathoracic anastomotic leaks following esophagogastrostomy and acute perforations. Segalin et al. and Roy-Choudhury et al. were among the first to report the successful treatment of an esophageal perforation or an anastomotic leak using a self-expanding metal stent, respectively $(16,17)$.

Several other authors reported their initial experiences treating acute perforations or anastomotic leaks between 2000 and 2005. Initial success rates in these series varied significantly as did the frequencies of stent migration, mortality and healing. The variability in results is not unexpected given the lack of treatment protocols among investigators, the evolutionary nature of the technique during this period and the diversity of stents used. Pleural drainage and enteral nutrition are noticeably absent as a consistent part of the treatment protocol.

Between 2005 and 2011, several series were reported containing at least ten acute perforation patients treated with esophageal stent placement. Johnsson et al. [2005] and Fischer and colleagues [2006] reported 20 and 15 esophageal perforation patients, respectively, treated with self-expanding metal stents. Johnsson reported a $95 \%$ sealing rate for the perforation but only a $77 \%$ rate of healing $(18,19)$. Fischer et al. realized a $100 \%$ rate of sealing the perforation and ultimate healing. Seven patients in this series developed an empyema requiring further intervention.

The utilization of esophageal stents in our practice began in high risk patients who had either previously undergone a failed operative repair or were unsuitable candidates for transthoracic repair of an esophageal leak. Our initial experience in 2007 found esophageal stents to be beneficial in these complex patients (20). In this series, silicone coated plastic stents were endoscopically placed in twenty one patients, all of whom had failed at least one operative repair for a chronic esophageal leak. The results were very encouraging; the success rate without further intervention was $95 \%$. In this early experience, we recognized the importance of optimizing nutritional status in this patient cohort as fourteen patients (67\%) underwent additional procedures for enteral feeding access.

The encouraging results of this initial investigation led us to query whether endoluminal esophageal stenting would be superior to primary operative repair in acute esophageal perforations. Recognizing the traditional goals of operative therapy for an esophageal perforation, we designed a hybrid treatment protocol which included operative or percutaneous drainage of infected spaces, the establishment of enteral nutrition along with esophageal stent placement. We also felt it was important for the thoracic surgeon to be involved in these patients care even when no surgery was required.

Our next report, also in 2007 analyzed the results of seventeen patients with an acute iatrogenic esophageal perforation treated using this hybrid strategy. Stent insertion, placement of percutaneous endoscopic gastrostomy tube and drainage of infected areas were achieved simultaneously in the operating room under general endotracheal anesthesia. Our results found that the perforation was sealed in 16 (94\%) patients with $82 \%$ resuming oral intake within seventy 2 hours (21). Stent migration occurred in three patients (18\%). Mean hospital length of stay was eight days (median 5 days) and there were 
no mortalities.

We then assessed the viability of using an esophageal stent in the treatment of a more difficult population of patients; those suffering a spontaneous esophageal perforation or Boerhave's syndrome and traditionally treated with operative strategies. Reported in 2009, this series of 19 patients found that 17 patients (89\%) sealed their perforation following stent placement (22). Fifteen patients $(79 \%)$ resumed oral intake within 72 hours of stent placement. Four patients $(21 \%)$ had at least one episode of stent migration requiring repositioning or replacement. Again, no mortalities resulted in this patient series. Results from this query have led to our increased confidence in utilizing esophageal stent placement even in patients who initially present with acute perforation and sepsis. In this same series, three patients (16\%) presented with findings consistent with sepsis and 13 patients (68\%) had clear evidence of mediastinitis on presentation. Effective stenting and rapid washout of the infected spaces allowed for a decrease in traditional operative time and increased efficiency for aggressive resuscitation.

During this time period, other centers were gaining experience treating esophageal perforation with stenting, as well. In 2009, Leers et al. reported a series of 15 patients treated with self-expanding metal stents, achieving a success rate of $87 \%$ without further intervention (23). Van Heel and colleagues also reported a series of 33 patients with an esophageal perforation treated with a variety of stent types in 2010 (24). Despite the stent sealing the perforation in 32 (97\%) patients, 4 (12\%) patients eventually required esophagectomy because of a recurrent leak or complications of removing the stent. In 2011, D'Cunha reported the University of Minnesota's experience with 37 patients, 15 of whom were treated for perforation (25). This study showed the value of a learning curve in the treatment of these patients and confirmed the hybrid approach of endoluminal stent placement with aggressive surgical drainage of infected spaces to be safe and effective in the treatment of acute esophageal perforation.

Over the last 5 years, the technique of esophageal stent placement for acute esophageal perforation has become more commonly utilized. As with any technique, the dissemination of this treatment strategy has allowed for continued innovation as well as some undesirable modifications. Ignoring the traditional goals of treatment for an esophageal perforation, some have chosen to use an esophageal stent as monotherapy for an esophageal perforation. Reports exist of multiple covered and uncovered stents being placed in patients with an esophageal perforation with continued mediastinal soilage without ever sealing the leak or draining infected spaces $(26,27)$. Such endeavors have predictably failed. Some investigators have also reported life threatening vascular fistulas following lengthy stent dwell times.

We sought to address each of these reported concerns in two investigations. The first was an analysis of stent failures. Reviewing 187 patients who underwent stent placement, four factors were associated with an increase rate of failure: (I) an injury greater than $6 \mathrm{~cm}$ in length; (II) injury that traversed the gastroesophageal junction or (III) proximal cervical esophagus or (IV) was associated with a leak in the gastric conduit in esophagectomy patients (28). Fifteen patients (8\%) who failed initial esophageal stent treatment were converted to traditional transthoracic (11 patients) or transabdominal (4 patients) operative repair at a mean of 3 days. This further emphasized the need to recognize stent treatment failure early and move to a traditional operative repair to avoid the complications of mediastinitis and need for esophagectomy or esophageal diversion. In addition, Persson et al. performed a 10 -year retrospective study reviewing the risk factors for esophageal stent failure when used as first line treatment. They concluded that continuous leakage after application of a stent, reduced preoperative physical performance, and concomitant esophagotracheal fistulas were independent risk factors. In their study, patients with severe mediastinitis, cervical anastomosis and conduit necrosis were excluded and underwent surgical intervention instead (29).

Our esophageal stenting experience was also reviewed in an attempt to determine the optimal time for esophageal stent removal (30). In our retrospective 7-year study, 162 patients were identified to have underwent esophageal stent placement for an acute perforation $(n=117)$ or anastomotic leak $(n=45)$. In the acute perforation cohort, stent removal in less than 28 days had an independent and significantly reduced complication rate (39\%). Patients whose stent was removed in less than 14 days after placement for an anastomotic leak had a stent complication rate that was also significant and independently reduced $(56 \%)$. We concluded that increased rates of complications were realized in patients whose stent was left in place for more than 4 weeks when treating an acute perforation or longer than 2 weeks when treating an anastomotic leak. Leaks which persisted beyond these time intervals were evaluated for surgical repair or a modification of stent therapy.

As mentioned, additional beneficial uses for the new 


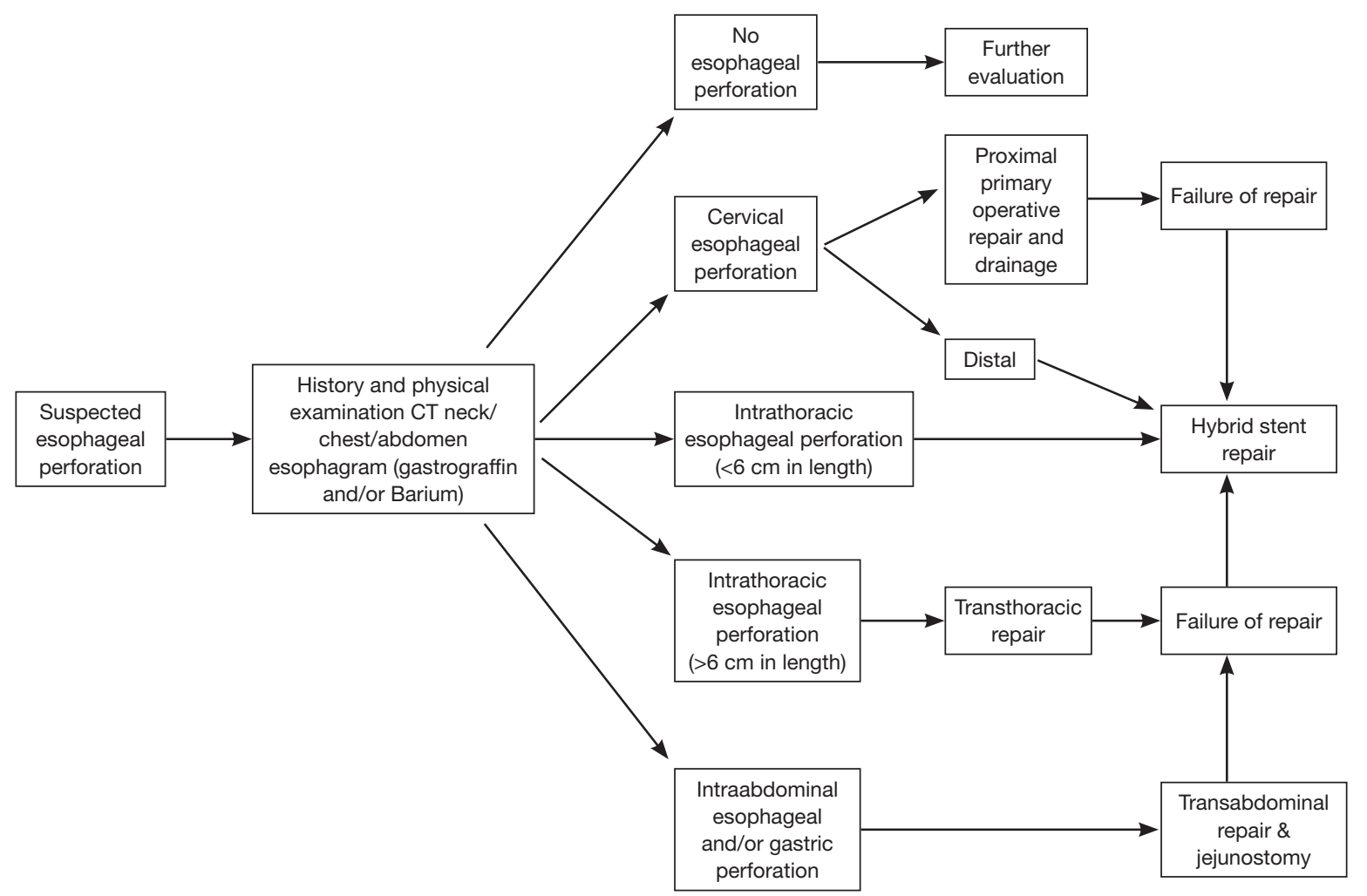

Figure 1 Treatment algorithm for esophageal perforation.

generation of esophageal stenting have been recognized as familiarity with the current generation of esophageal stents has increased. Intrathoracic anastomotic leak following esophagogastrostomy remains a morbid complication of esophagectomy. However several groups have shown that esophageal stent placements for such leaks are safe and effective treatment while avoiding reoperation or diversion in the vast majority of cases. Our experience with a series of seventeen patients reported successful sealing of all anastomotic leaks with the placement of esophageal stents. The majority of who previously received preoperative chemotherapy and radiation therapy (31). From this series, three patients underwent further treatment, namely, endoscopic removal and replacement for stent migration (18\%).

Keeling and colleagues, when reporting their results in primary surgical repair of esophageal perforation patients, discussed their successful novel use of an esophageal stent in two patients whose leak persisted (32). We subsequently reviewed our experience in patients treated with surgical repair who continued to experience a significant leak (33). In a series of 29 such patients, esophageal stent placement was able to "rescue" the initial repair and allowed healing in $27(93 \%)$ patients without further operative intervention. Twenty-five (86\%) patients tolerated oral feeds within 72 hours of stent placement. Mean hospital length of stay was 8 days.

\section{Current state}

Our current practice considers esophageal stent placement for any esophageal perforation, fistula or anastomotic leak (Figure 1). Duration of the perforation or fistula prior to treatment, whether a previous operative repair has been performed are irrelevant. We also consider patients who have a relatively large esophageal diameter or patients who present with systemic manifestations of infection related to their esophageal injury.

In regards to esophageal malignancy, we have found that acute perforations or fistulae can be successfully treated and often seal with endoluminal stent placement. Thus, temporizing the patient without the need to proceed towards an esophagectomy. Systemic chemotherapy and/ or radiation therapy is discontinued for a 2 -week period but 


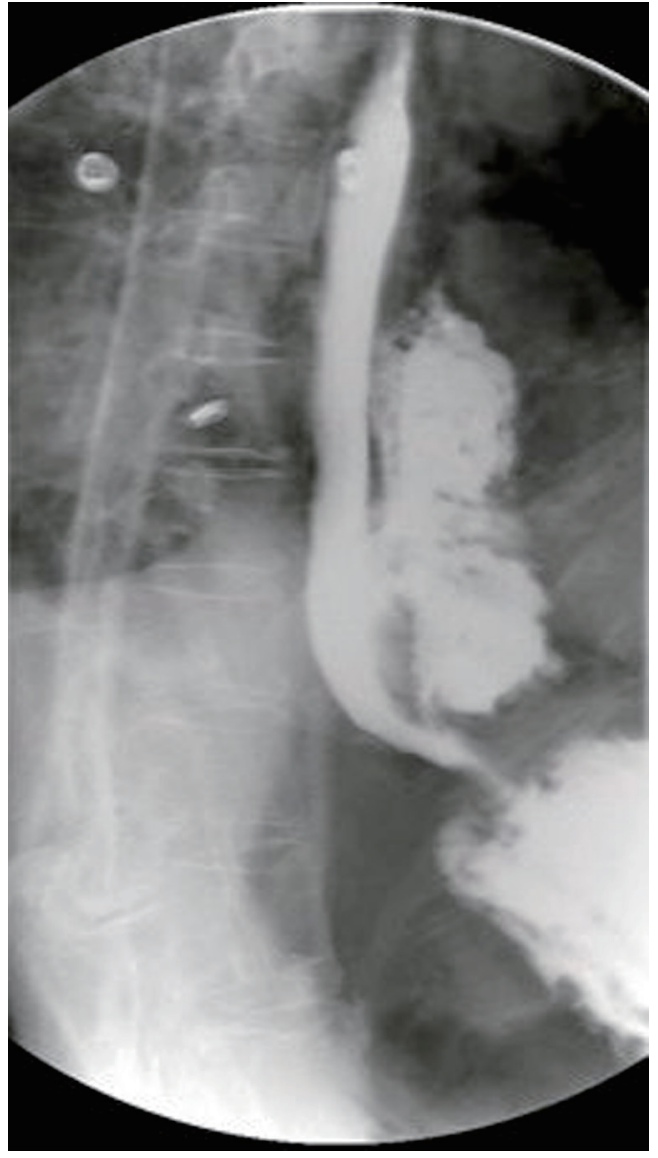

Figure 2 Contrast esophagram displaying a mid-thoracic esophageal perforation.

can then be restarted.

\section{Exclusion criteria}

As with any technique, there are some relative contraindications to this form of treatment. Long segment perforations of the esophagus $(>6 \mathrm{~cm})$, esophageal injuries recognized during another operative procedure or in a patient that will require an immediate thoracotomy for an associated injury are generally treated with a traditional operative repair. Anatomically, acute cervical esophageal injuries are better treated with operative repair as most patients are unable to tolerate a stent that lies proximal to the cricopharyngeus muscle. However, in select patients, we have seen successful esophageal stent treatment of chronic cervical esophageal fistulae.

In the case of an intrathoracic anastomotic leak following esophagectomy, a near complete dehiscence of the anastomosis or focal or generalized conduit necrosis are not treated by esophageal stent placement for obvious reasons. Similarly, esophagectomy patients whose foregut continuity was reestablished with a conduit other than the stomach who experience an anastomotic leak have not undergone esophageal stent placement in our practice. The endoluminal stent size required to seal the larger caliber esophageal side of an anastomotic leak can result in necrosis of a jejunal conduit while the potential for significant complications related to stent migration is increased if the colon was used for reconstruction.

\section{Stent placement technique}

Prior to intervention, a Gastrograffin and/or Barium esophagram is obtained to localize presence of an esophageal perforation, fistula or anastomotic leak (Figure 2). Intervention is warranted when a significant leak is documented, namely extravasation of contrast from the lumen of the esophagus into the mediastinum, pleural or peritoneal space. To identify other areas of secondary infection requiring further management and/or surgical drainage, all patients undergo computer-aided tomographic imaging of the neck, chest and abdomen (Figure 3).

All esophageal stents are placed in the operating room using general anesthesia and fluoroscopy by a thoracic surgeon following flexible esophagoscopy. The purposes of esophagoscopy are to localize the esophageal leak and decide which size stent should be utilized (Figure 4). Choice of stent is by surgeon discretion; in our institution we utilize a mix of fully covered, self-expanding plastic or metal stents. Our approach has been to oversize the stent both in length and diameter. This has had a beneficial effect on stent migration and, we believe, the ability to seal a leak relatively quickly. We generally use a 21 to 25 millimeters diameter stent in the longest length possible without crossing the gastroesophageal junction or the arytenoid fold of the posterior oropharynx. Placement of the stent is centered on the perforation area with ample length on either proximal or distal ends. We have not found it beneficial to routinely place more than one stent in the esophagus in an attempt to seal a perforation, fistula or anastomotic leak. In our practice, it is also important to establish enteral access prior to stent placement. If a patient has not undergone esophagectomy or has no other form of enteral access, a percutaneous endoscopic gastrostomy or laparoscopic jejunostomy tube is performed. Both can be placed to gravity drainage initially negating the need for a nasogastric 


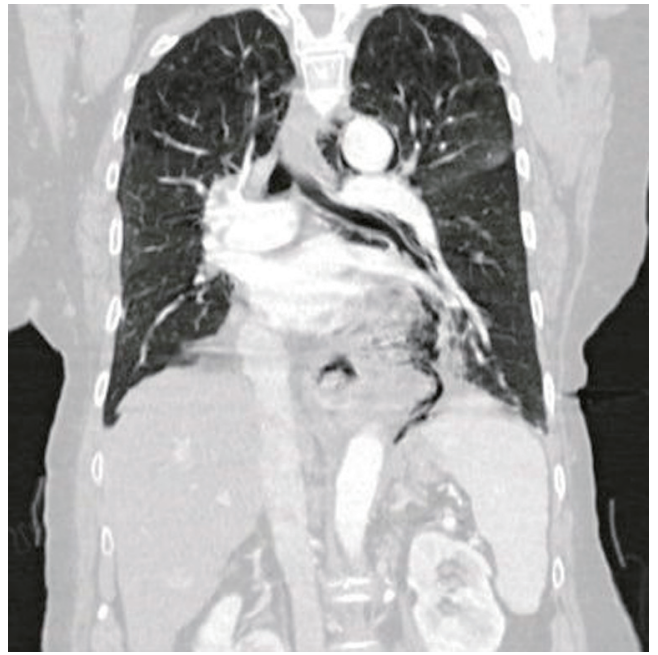

Figure 3 Computer aided tomographic imaging of the chest following an esophageal perforation.

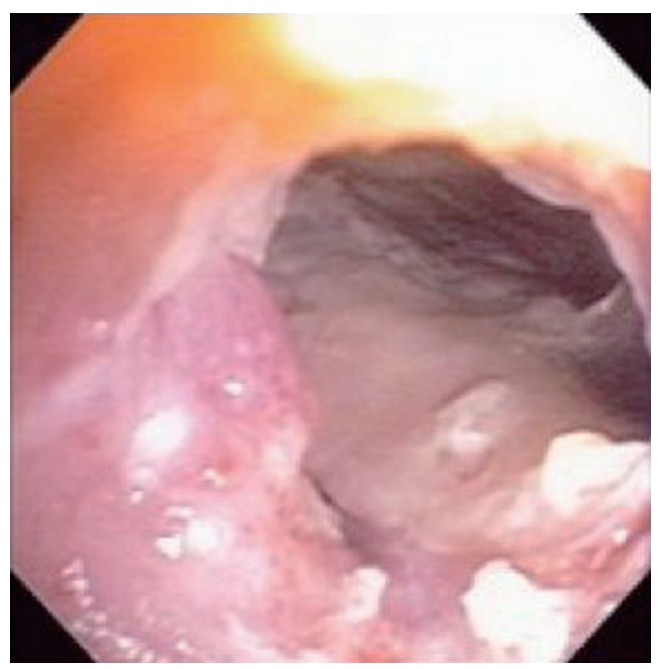

Figure 4 Endoscopic view of an acute esophageal perforation.

tube with feedings thereafter.

Proximal and distal landmarks are marked with a radiopaque object and while utilizing fluoroscopy for stent deployment (Figure 5). A guide wire is inserted through the endoscope, followed by the stent which is threaded through under direct fluoroscopy, positioned and deployed. Repeat esophagoscopy is utilized to confirm proper placement of the stent

In case adjustments should be made, the stent can be easily grasped and moved to a more proximal point using endoscopy and/or fluoroscopy. However, if the stent is too

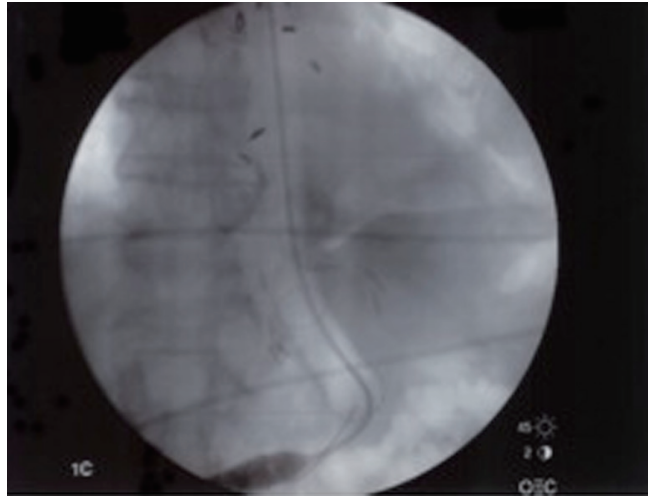

Figure 5 Fluoroscopic image of an esophageal stent placement for acute perforation.

proximal after deployment, it will likely need to be removed and replaced to prevent further injury to the esophagus. If the stent does not open completely after its deployment, a pneumatic esophageal dilatation balloon can be used to expand it. Within 24 hours, the stent tends to fully expand without further intervention and lies snug against the esophagus.

We have found that endoscopy following stent placement is a better assessment of stent placement and deployment (Figure 6). We do not routinely perform a contrast study at the time of stent placement. Our preference has been to perform a contrast esophagram 48 to 72 hours post stent placement as it takes the stent some time to equilibrate to body temperature, maximally expand and form a proper seal.

Importantly, as part of the hybrid protocol, adequate drainage of infected areas is achieved during the same anesthesia either by video-assisted thoracoscopy, laparoscopy or image-guided percutaneous drainage. Such procedures are planned based on the preoperative imaging studies and findings at the time of endoscopy. We do no routinely perform a tracheostomy in these patients unless they have already required prolonged mechanical ventilation to reduce the possibility of tracheoesophageal fistula formation with prostheses in both the esophagus and trachea.

As with many surgical procedures, the care of the patient following esophageal stent placement is just as important as the performance of the procedure itself. We feel it is important that the thoracic surgeon direct the care of these patients just as if they had undergone an operative repair. This is not only important in making sure patients who 


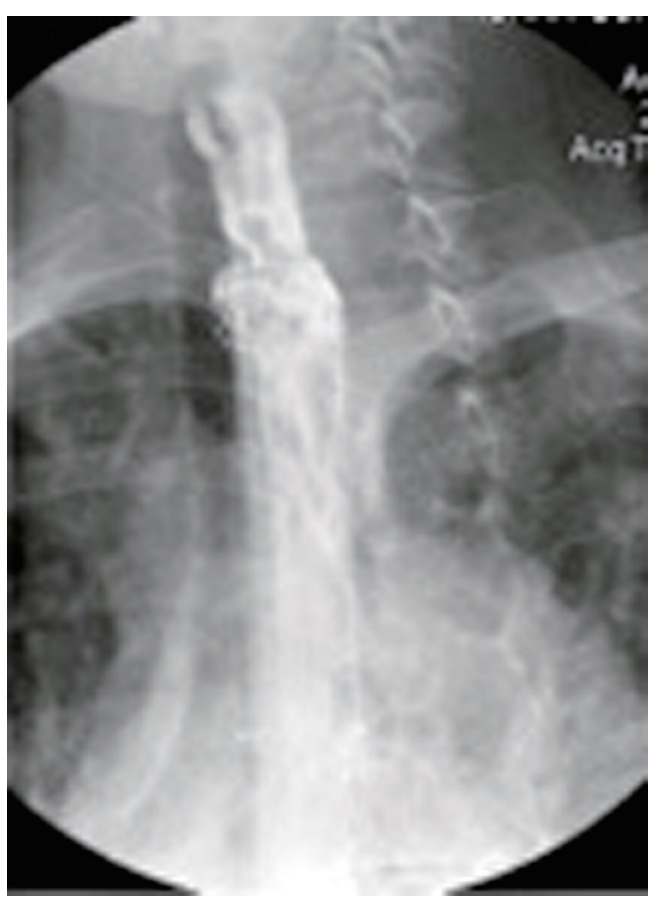

Figure 6 Esophagram 48 hours after esophageal stent placement for acute perforation.

fail stent therapy receive timely operative intervention, but is essential in determining that all associated areas of intrathoracic or intraabdominal infection are adequately treated.

Similarly, the systemic inflammatory response and/or sepsis experienced by some of these patients require that they be cared for with the same vigilance as if they had undergone an operative repair. This includes appropriate fluid resuscitation, prophylactic antimicrobial therapy adjusted by culture data and cardiopulmonary support administered in a critical care environment. Especially important is the immediate initiation of nutrition; preferably via an enteral route.

Patients with persistent or recurrent fevers, organ failure or leukocytosis undergo computer aided tomographic imaging of the neck, chest and abdomen every 48 to 72 hours to look for evidence of surgically treatable infection (6). If identified, such infection must be aggressively treated. It is our preference to perform such procedures in a minimally invasive fashion, when possible.

Contrast esophagram is performed at minimum after 24 hours after stent placement to document successful leak occlusion (Figure 5). A "mechanical soft" diet is then initiated, excluding bread and meat to minimize stent migration. Enteral feeding is continued until the patient can consume sufficient calories to maintain their nutritional parameters.

Initiation of an oral diet should be paired with a daily proton pump inhibitor and multivitamins to maximize healing. Aspiration precautions are evaluated with a bedside swallow study prior to starting oral diet. Post procedure pain is usually managed with a patient controlled analgesia and a short acting opioid. Patients are usually discharged with an oral narcotic elixir, if required. Notably, we have not removed stents placed for perforation, fistula or anastomotic leak secondary to intractable pain. This is in contrast to the rare patient with benign disease who experiences severe pain warranting stent removal.

As previously alluded to, stent migration does occur with esophageal stent placement, techniques to aid minimizing the occurrence and trouble-shoot have been described. Initial management should include chest roentogram followed by endoscopy in the operating room as soon as possible. During endoscopy, the site of the esophageal leak or injury should be carefully inspected as migration can be a sign that the leak has been sealed. We do not exchange an esophageal stents unless migration not amenable to repositioning has occurred or if the integrity of the stent is compromised.

It is the intention to remove all patients' esophageal stents following a sufficient amount of time to allow the leak to seal. This is individualized for each patient taking into consideration the resolution of any indications of systemic infection. Stent removal is also carried out in the operating room under general anesthesia. Flexible esophagoscopy is performed before and after stent removal and an esophagram is performed before oral intake is resumed.

Our experience has led us to remove stents much earlier than we did in our initial series. This is due to a better understanding of how these leaks seal and then heal; adherence to adjacent structures creating a watertight seal followed by eventual tissue in-growth and repair. Several reports of significant complications related to indwelling esophageal stents including tracheo-esophageal fistulae, aorto-esophageal fistulae and bowel obstruction resulting from stent migration have also prompted us to routinely consider stent removal after the earliest duration of 10 to 14 days up to 4 weeks.

Any measure of success in treating patients with an esophageal injury that we have obtained stems from our adherence to several basic principles: (I) management directed by a thoracic surgeon; (II) early diagnosis; (III) 
confirmation of sealing of the esophageal discontinuity after stent placement; (IV) drainage of pleura cavity with simultaneous PEG tube for early enteral nutrition; and (V) early intravenous antibiotic coverage. All of the above are simultaneous while the patient is managed in the intensive care unit.

The thoracic surgeon must be involved in the care decisions for patients with esophageal perforation. This is important both to identify contraindications for stent placement, in which immediate operative repair is undertaken, and to rapidly identify and treat stent failures. It is also important to ensure that these patients receive the level of care their condition requires, including aggressive antimicrobial and nutritional therapy, surveillance for continued areas of infection, and critical care support.

\section{Outcomes}

Any analysis of the effectiveness of esophageal stent placement must be made in the context of the application of the technique and in comparison to the available treatment alternatives. The authors feel strongly that esophageal stent placement as monotherapy for esophageal perforation is inadequate and does not recognize the traditional goals of therapy for a patient with an esophageal perforation; a principle confirmed by a recent literature review by Dasari and colleagues (34). Licht et al. in their 2016 retrospective publication ( $\mathrm{n}=49)$ also affirmed that combination of stent placement, enteral tube feeding for nutritional support, combined with drainage was highly effective in achieving healing. A substantial portion (82\%) received a jejunostomy tube with 95\% successful anastomotic healing (35). Therefore it is not surprising to see results in some series or isolated case reports which are not comparable to a hybrid treatment protocol that emphasizes drainage of infected spaces, enteral nutrition and early recognition of persistent leak with stent revision or operative repair.

Despite advances in surgical technique, critical care and antimicrobial therapy, surgical repair continues to be associated with a postoperative leak rate reported between $30 \%$ and $40 \%$ at experienced centers $(32,36)$. The reported rate of successful stent use in esophageal perforation is at least comparable if not superior to these results without the need for thoracotomy. Stent use also provides a rescue mechanism for failed operative repair and has nearly eliminated the need for esophagectomy or esophageal diversion in our practice. Stent use may also reduce the incidence of long term esophageal stricture when compared to operative repair. In current literature, use of esophageal stenting have also been extrapolated across various esophageal diseases as recently reported in a multicenter, retrospective publication on a cohort of seventy patients (37). Success rates were high across the board in treatment of acute perforations (100\%), fistula (71.4\%) and anastomotic leaks (80\%). In comparison, refractory benign esophageal strictures $(33.3 \%)$ and anastomotic strictures (23.1\%) had lower success rates. The authors perceived no differences in superiority of various types of stents. Speer et al. in their 10-year retrospective review also reported higher success with treatment of leaks $(70 \%)$ versus strictures (27\%) (38).

Like any technique, esophageal stent use for acute perforation does have its associated morbidities. The principle complication associated with stent use remains stent migration (38). The use of an esophageal stent also commits the patient to a return to surgery for stent removal and/or revision if migration does occur. Lastly, rarely reported fatal complications have been reported from vascular fistulae with what are now considered excessive stent dwell times.

It is doubtful for several reasons that a randomized trial between stent placement and operative repair for acute esophageal perforation will occur in the near future, not the least of which is the possible lack or equipoise between the two patient cohorts based on recent literature. However comparisons can be made between the two treatment groups. In 2014, we reported a propensity matched comparison of patients treated at multiple institutions with either an esophageal stent or an operative repair following the recognition of an acute esophageal perforation (39). In these well matched groups, the esophageal stent cohort realized significant differences in morbidity ( $4 \% v s .43 \%$; $\mathrm{P}=0.02)$, mean length of stay ( 6 vs. 11 days; $\mathrm{P}=0.0007)$, time to oral intake ( 3 vs. 8 days; $\mathrm{P}=0.0004)$, and cost $(\$ 91,000$ vs. $\$ 142,000 ; \mathrm{P}<0.0001)$ were identified in the esophageal stent cohort when compared with patients receiving surgical repair. Ben-David et al. reported similar findings in a recently published single center experience (40).

The continued success of the concept of treating an esophageal perforation without surgical repair has led to further development of other innovative endoluminal approaches to these disorders. Direct endoluminal closures of iatrogenic perforations have been reported with clips (through-the-scope, over-the-scope) and endoluminal suturing techniques (41-43). A recent metaanalysis reviewed current literature reporting on endoclip 
technique in closure of iatrogenic perforations. The authors concluded that use of endoclips in the esophagus could be considered when perforation diameter is small (less than $2 \mathrm{~cm}$ ) but recommend surgical intervention if perforation is recognized late (greater 24 hours), or larger perforation diameter $(>2 \mathrm{~cm})$ and if there is evidence of leak into the mediastinum (44). Verlaan et al. performed a large metaanalysis on various endoscopic closure systems. They authors reported successful closure with endoclips (90.2\%) and over-the-scope clips $(87.8 \%)$ but also concluded that there remains many limitations in current literature including the lack of randomized control trials and low methodological quality of studies (45). Wound vacuum therapy has also been investigated and shows promise, especially in patients with an esophageal fistula (46). A single-center retrospective study by Mennigen et al. compared 30 patients who underwent endoscopic stent placement to fifteen patients with vacuum therapy. Though a small study, their findings were promising with high success rates for vacuum therapy (47). With the increased popularity in Europe (Germany) and growing interest in the US, a recent case series by Smallwood et al. was reported on six patients with esophageal perforations who were treated with a modified E-vac therapy. All six had successful closure of their esophageal perforation (average duration 35.8 days) as evidenced on both esophagoscopy and CT imaging (48). Alone, and in combination with esophageal stent placement, these techniques provide options for patients with complex esophageal injuries and leaks.

\section{Conclusions}

Over the past decade, the experience and utilization of endoluminal esophageal stents both at our center and elsewhere has exponentially increased. In combining traditional treatment goals with stent technology, we have developed a hybrid protocol for treatment of esophageal perforations, fistulae and leak with good results. While we have been pleased with the results of endoluminal esophageal stent placement for the treatment of esophageal injuries, there are significant questions which remain to be answered. These include comparing different types of stents in a prospective fashion and assessing the results of this technique in multiple centers using a common treatment protocol. There also remain multiple areas of improvement, including reassessment of current protocols and prevention of notable complications such as stent migration, stent erosion, trachea-esophageal or aorto-esophageal fistulae.
It is our belief that with continued investigation and refinement, esophageal stent placement will become a more integral part of the thoracic surgeons' armamentarium when caring for patients with acute and chronic diseases of the esophagus.

\section{Acknowledgements}

None.

\section{Footnote}

Conflicts of Interest: The authors have no conflicts of interest to declare.

\section{References}

1. Symonds CJ. The treatment of malignant stricture of the oesophagus by tubage or permanent catheterism. Br Med J 1887;1:870-3.

2. Guisez J. De l'intubation caouchoutee oesophienne. Presse Med 1914;22:85.

3. Souttar HS. A method of intubating the esophagus for malignant stricture. Br Med J 1924;1:782-3.

4. Coyas A. Palliative intubation in carcinoma of the oesophagus. Lancet 1955;269:647-9.

5. Mousseau M, Le Forestier J, Barbin J. Role of permanent intubation in palliative treatment of esophageal cancer. Arch Mal Appar Dig Mal Nutr 1956;45:208-14.

6. Atkinson M, Ferguson R, Ogilvie AL. Management of malignant dysphagia by intubation at endoscopy. J R Soc Med 1979;72:894-7.

7. Celestin LR. Permanent intubation in inoperable cancer of the oesophagus and cardia: a new tube. Ann R Coll Surg Engl 1959;25:165-70.

8. Chan AC, Shin FG, Lam YH, et al. A comparison study on physical properties of self-expandable esophageal metal stents. Gastrointest Endosc 1999;49:462-5.

9. Schmassmann A, Meyenberger C, Knuchel J, et al. Self-expanding metal stents in malignant esophageal obstruction: a comparison between two stent types. Am J Gastroenterol 1997;92:400-6.

10. Ell C, Hochberger J, May A, et al. Coated and uncoated self-expanding metal stents for malignant stenosis in the upper GI tract: preliminary clinical experiences with Wallstents. Am J Gastroenterol 1994;89:1496-500.

11. Nelson DB, Silvis S, Ansel H. Management of a tracheoesophageal fistula with a silicone-covered 
self-expanding metal stent. Gastrointest Endosc 1994;40:497-9.

12. Ramirez FC, Dennert B, Zierer ST, et al. Esophageal self-expandable metallic stents--indications, practice, techniques, and complications: results of a national survey. Gastrointest Endosc 1997;45:360-4.

13. Saxon RR, Morrison KE, Lakin PC, et al. Malignant esophageal obstruction and esophagorespiratory fistula: palliation with a polyethylene-covered Z-stent. Radiology 1997;202:349-54.

14. Decker P, Lippler J, Decker D, et al. Use of the Polyflex stent in the palliative therapy of esophageal carcinoma: results in 14 cases and review of the literature. Surg Endosc 2001;15:1444-7.

15. Gelbmann CM, Ratiu NL, Rath HC, et al. Use of selfexpandable plastic stents for the treatment of esophageal perforations and symptomatic anastomotic leaks. Endoscopy 2004;36:695-9.

16. Segalin A, Bonavina L, Lazzerini M, et al. Endoscopic management of inveterate esophageal perforations and leaks. Surg Endosc 1996;10:928-32.

17. Roy-Choudhury SH, Nicholson AA, Wedgwood KR, et al. Symptomatic malignant gastroesophageal anastomotic leak: management with covered metallic esophageal stents. AJR Am J Roentgenol 2001;176:161-5.

18. Johnsson E, Lundell L, Liedman B. Sealing of esophageal perforation or ruptures with expandable metallic stents: a prospective controlled study on treatment efficacy and limitations. Dis Esophagus 2005;18:262-6.

19. Fischer A, Thomusch O, Benz S, et al. Nonoperative treatment of 15 benign esophageal perforations with self-expandable covered metal stents. Ann Thorac Surg 2006;81:467-72.

20. Freeman RK, Ascioti AJ, Wozniak TC. Postoperative esophageal leak management with the Polyflex esophageal stent. J Thorac Cardiovasc Surg 2007;133:333-8.

21. Freeman RK, Van Woerkom JM, Ascioti AJ. Esophageal stent placement for the treatment of iatrogenic intrathoracic esophageal perforation. Ann Thorac Surg 2007;83:2003-7.

22. Freeman RK, Van Woerkom JM, Vyverberg A, et al. Esophageal stent placement for the treatment of spontaneous esophageal perforations. Ann Thorac Surg 2009;88:194-8.

23. Leers JM, Vivaldi C, Schafer H, et al. Endoscopic therapy for esophageal perforation or anastomotic leak with a selfexpandable metallic stent. Surg Endosc 2009;23:2258-62. 24. van Heel NC, Haringsma J, Spaander MC, et al. Short- term esophageal stenting in the management of benign perforations. Am J Gastroenterol 2010;105:1515-20.

25. D'Cunha J, Rueth NM, Groth SS, et al. Esophageal stents for anastomotic leaks and perforations. J Thorac Cardiovasc Surg 2011;142:39-46.e1.

26. Odell JA, DeVault KR. Extended stent usage for persistent esophageal leak: should there be limits? Ann Thorac Surg 2010;90:1707-8.

27. Whitelocke D, Maddaus M, Andrade R, et al. Gastroaortic fistula: a rare and lethal complication of esophageal stenting after esophagectomy. J Thorac Cardiovasc Surg 2010;140:e49-50.

28. Freeman RK, Ascioti, AJ, Giannini T, et al. An Analysis of Unsuccessful Esophageal Stent Placements for Esophageal Perforation, Fistula or Anastomotic Leak. Ann Thorac Surg 2012;94:959-64.

29. Persson S, Rouvelas I, Kumagai K, et al. Treatment of esophageal anastomotic leakage with self-expanding metal stents: analysis of risk factors for treatment failure. Endosc Int Open 2016; 4:E420-6.

30. Freeman RK, Ascioti, AJ, Dake M, et al. An Assessment of the Optimal Time for Removal of Esophageal Stents used in the Treatment of an Esophageal Anastomotic Leak or Perforation. Ann Thorac Surg 2015:100;422-8.

31. Freeman RK, Vyverberg A, Ascioti AJ. Esophageal stent placement for the treatment of acute intrathoracic anastomotic leak after esophagectomy. Ann Thorac Surg 2011;92;204-8.

32. Keeling WB, Miller DL, Lam GT, et al, Low mortality after treatment for esophageal perforation: a single-center experience. Ann Thorac Surg 2010;90:1669-73; discussion 1673.

33. Freeman RK, Ascioti AJ, Dake M, et al. An analysis of esophageal stent placement for persistent leak after the operative repair of intrathoracic esophageal perforations. Ann Thorac Surg 2014;97:1715-9; discussion 1719-20.

34. Dasari BV, Neely D, Kennedy A, et al, The role of esophageal stents in the management of esophageal anastomotic leaks and benign esophageal perforations. Ann Surg 2014;259:852-60.

35. Licht E, Markowitz A, Bains M, et al. Endoscopic Management of esophageal anastomotic leaks after surgery for malignant disease. Ann Thorac Surg 2016;101:301-4.

36. Wright CD, Mathisen DJ, Wain JC, et al. Reinforced Primary Repair of Thoracic Esophageal Perforation. Ann Thorac Surg 1995;60:245-8.

37. Suzuki T, Siddiqui A, Taylor L, et al. Clinical outcomes, efficacy and adverse events in patients undergoing 
esophageal stent placement for benign indications: a large multicenter study. J Clin Gastroenterol 2016;50:373-8.

38. Speer E, Dunst C, Shada A, et al. Covered stents in cervical anastomoses following esophagectomy. Surg Endosc 2016;30;3297-303.

39. Freeman RK, Herrera A, Ascioti AJ, et al. A PropensityMatched Comparison of Cost and Outcomes following Esophageal Stent Placement or Primary Surgical Repair for Esophageal Perforation. J Thorac Cardiovasc Surg 2015;149:1550-5.

40. Ben-David K, Behrns K, Hochwald S, et al, Esophageal perforation management using a multidisciplinary minimally invasive treatment algorithm. J Am Coll Surg 2014;218:768-74.

41. Raju GS. Endoscopic closure of gastrointestinal leaks. Am J Gastroenterol 2009;104:1315-20.

42. Kirschniak A, Kratt T, Stüker D, et al. A new endoscopic over-the-scope clip system for treatment of lesions and bleeding in the GI tract: first clinical experiences. Gastrointest Endosc 2007;66:162-7.

43. Baron TH, Wong Kee Song LM, Zielinski MD, et

Cite this article as: Ong GK, Freeman RK. Endoscopic management of esophageal leaks. J Thorac Dis 2017;9(Suppl 2): S135-S145. doi: 10.21037/jtd.2017.03.100 al. A comprehensive approach to the management of acute endoscopic perforations. Gastrointest Endosc 2012;76:838-59.

44. Yılmaz B, Unlu O, Roach EC, et al. Endoscopic clips for the closure of acute iatrogenic perforations: Where do we stand? Dig Endosc 2015;27:641-8.

45. Verlaan T, Voermans RP, van Berge Henegouwen MI, et al. Endoscopic closure of acute perforations of the GI tract: a systematic review of the literature. Gastrointest Endosc 2015;82:618-28.e5.

46. Schorsch T, Müller C, Loske G. Endoscopic vacuum therapy of anastomotic leakage and iatrogenic perforation in the esophagus. Surg Endosc 2013;27:2040-5.

47. Mennigen R, Harting C, Lindner K, et al. Comparison of Endoscopic Vacuum Therapy Versus Stent for Anastomotic Leak After Esophagectomy. J Gastrointest Surg 2015;19:1229-35.

48. Smallwood NR, Fleshman JW, Leeds SG, et al. The use of endoluminal vacuum (E-Vac) therapy in the management of upper gastrointestinal leaks and perforations. Surg Endosc 2016;30:2473-80. 